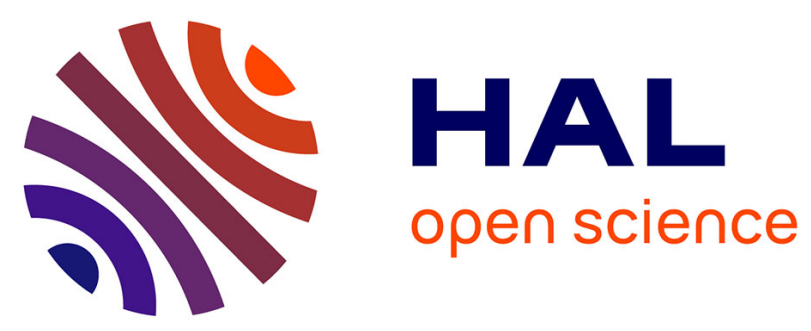

\title{
Melting curve and chemical stability of ammonia at high pressure: a combined x-ray diffraction and Raman study
}

Jean-Antoine Queyroux, Sandra Ninet, Gunnar Weck, Gaston Garbarino, Thomas Plisson, Mohamed Mezouar, Frédéric Datchi

\section{- To cite this version:}

Jean-Antoine Queyroux, Sandra Ninet, Gunnar Weck, Gaston Garbarino, Thomas Plisson, et al.. Melting curve and chemical stability of ammonia at high pressure: a combined x-ray diffraction and Raman study. Physical Review B: Condensed Matter and Materials Physics (1998-2015), 2019, 99 (13), pp.134107. 10.1103/PhysRevB.99.134107 . hal-02287302

\section{HAL Id: hal-02287302 https://hal.sorbonne-universite.fr/hal-02287302}

Submitted on 13 Sep 2019

HAL is a multi-disciplinary open access archive for the deposit and dissemination of scientific research documents, whether they are published or not. The documents may come from teaching and research institutions in France or abroad, or from public or private research centers.
L'archive ouverte pluridisciplinaire HAL, est destinée au dépôt et à la diffusion de documents scientifiques de niveau recherche, publiés ou non, émanant des établissements d'enseignement et de recherche français ou étrangers, des laboratoires publics ou privés. 


\title{
Melting curve and chemical stability of ammonia at high pressure: a combined x-ray diffraction and Raman study
}

\author{
Jean-Antoine Queyroux, ${ }^{1, *}$ Sandra Ninet, ${ }^{1}$ Gunnar Weck, ${ }^{2}$ Gaston \\ Garbarino, ${ }^{3}$ Thomas Plisson, ${ }^{2}$ Mohamed Mezouar, ${ }^{3}$ and Frédéric Datchi ${ }^{1, \dagger}$ \\ ${ }^{1}$ Institut de Minéralogie, de Physique des Matériaux et de Cosmochimie (IMPMC), Sorbonne Université, \\ CNRS UMR 7590, IRD UMR 206, MNHN, 4 place Jussieu, F-75005 Paris, France \\ ${ }^{2}$ CEA, DAM, DIF, F-91297 Arpajon, France \\ ${ }^{3}$ European Synchrotron Radiation Facility, Boîte Postale 220, 38043 Grenoble, France
}

(Dated: April 4, 2019)

\begin{abstract}
The melting curve and stability of ammonia $\left(\mathrm{NH}_{3}\right)$ is investigated up to $40 \mathrm{GPa}$ and $3500 \mathrm{~K}$ by x-ray diffraction and Raman spectroscopy in the laser-heated diamond anvil cell. The $\mathrm{NH}_{3}$ samples were directly heated by the $10.6 \mu \mathrm{m}$ radiation of a $\mathrm{CO}_{2}$ laser to reduce the risks of chemical reactions. Melting was unambiguously detected by the appearance of the liquid diffraction signal upon temperature increase. The melting temperature of $\mathrm{NH}_{3}$ is found to steadily increase with pressure up to $40 \mathrm{GPa}$, and the previously reported turnover is not observed. As a result, the melting line of $\mathrm{NH}_{3}$ is expected to cross the isentropes of Neptune and Uranus in the pressure range 55-65 GPa, implying the possible presence of superionic solid $\mathrm{NH}_{3}$ in these planets. Our x-ray and Raman measurements confirm the appearance of $\mathrm{N}_{2}$ and $\mathrm{H}_{2}$ upon heating the liquid phase from 6 to $40 \mathrm{GPa}$. But while the equilibrium $2 \mathrm{NH}_{3} \rightleftharpoons \mathrm{N}_{2}+3 \mathrm{H}_{2}$ balances towards the dissociated elements at low pressure and high temperature, ammonia is found to the more stable species in the range 20-40 GPa, 300-3000 K.
\end{abstract}

PACS numbers: Valid PACS appear here

\section{INTRODUCTION}

Ammonia has a significant abundance in the outer solar system and, together with water and methane, is thought to be a major component of the ice layer of the giant planets Neptune and Uranus, of satellites such as Titan and Ganymede, and of some recently discovered exoplanets such as GJ $436 \mathrm{~b}$ or HAT-P-11b $\mathrm{b}^{1-5}$. Depending on the size of these bodies, the pressure $(\mathrm{P})$ at the bottom of the ice layer may range from a few to several hundreds of GPa, which makes the knowledge of the properties of these ices, such as the melting curve, equation of state and chemical stability, over a broad range of pressure, important input for modelling the icy planets.

The presently accepted phase diagram of ammonia is shown in Fig. 1. Below $4 \mathrm{GPa}$, three different structures have been experimentally evidenced. In increasing order of temperature $(\mathrm{T})$, these are the proton-ordered cubic solid $\mathrm{I}^{6-8}$, the plastic hexagonal close-packed solid $\mathrm{II}^{9}$ and the plastic face-centered cubic solid $\mathrm{III}^{10}$. From 4 to $\sim 60 \mathrm{GPa}$, the solid is either in the proton-ordered orthorhombic solid $\mathrm{IV}^{11}$ - which transforms to the isosymetric $\mathrm{V}$ above $12 \mathrm{GPa}^{12}$ at low temperature, or in phase III at high temperature. All these phases are molecular solids. Recent works have shown that above 120 to 150 $\mathrm{GPa}$ at $300 \mathrm{~K}$, the molecular solid V becomes unstable and $\mathrm{NH}_{3}$ transforms into an ionic structure of orthorhombic symmetry composed of $\mathrm{NH}_{2}^{-}$and $\mathrm{NH}_{4}^{+}$ions ${ }^{13,14}$. A second non-molecular phase has been evidenced at $\mathrm{P}$ $\mathrm{T}$ conditions beyond $57 \mathrm{GPa}-710 \mathrm{~K}$, which is a superionic conductor ${ }^{15}$. In the latter, protons rapidly diffuse through the lattice of nitrogen atoms via jumps along the $\mathrm{N}-\mathrm{H} \cdots \mathrm{N}$ hydrogen bonds. The existence of this su- perionic solid was previously predicted by DFT-based molecular dynamics simulations ${ }^{16}$ above $\sim 1200 \mathrm{~K}$, but the more recent theoretical studies of Refs. 15 and 17 are fully consistent with the experimental transition line.

The properties of the ammonia fluid have been so far mainly explored by shock-wave experiments which reported pressure-compression and electrical conductivity data in the range $\sim 2-65 \mathrm{GPa}, 1100-4600 \mathrm{~K}^{18-22}$. As for water, the electrical conductivity of fluid ammonia increases rapidly with the shock pressure up to $30 \mathrm{GPa}$ and then saturates at about $20 \Omega^{-1} \cdot \mathrm{cm}^{-1}$ at $60 \mathrm{GPa}$. This was interpreted as resulting from a rapid ionization of the fluid with pressure and temperature increase. The $a b$ initio molecular dynamics (AIMD) simulations of Cavazzoni et al. ${ }^{16}$ support the presence of this ionic fluid above $50 \mathrm{GPa}$ and $2000 \mathrm{~K}$, and predict the closure of the electronic band gap at $300 \mathrm{GPa}$ and $\sim 5500 \mathrm{~K}$. The more recent simulations of Bethkenhagen et al. ${ }^{17}$ predict three fluid domains: a molecular $\mathrm{NH}_{3}$ fluid $(P<30 \mathrm{GPa}$ and $T<4000 \mathrm{~K}$ ), a fluid mainly composed of diatomic $\mathrm{H}_{2}$ and $\mathrm{N}_{2}$ forming from the dissociation of $\mathrm{NH}_{3}(P<20 \mathrm{GPa}$ and $4000<T<5000 \mathrm{~K}$ ) and a fluid referred as "dissociated" but whose composition was not clearly defined $(20<P<350 \mathrm{GPa}$ and $2500<T<10000 \mathrm{~K})$.

There exists only few experimental investigations of the melting curve of ammonia above $300 \mathrm{~K}$. In 1980 , Hanson and Jordan ${ }^{23}$ reported measurements up to 373 $\mathrm{K}$ and $2 \mathrm{GPa}$. In 2008, Ninet and Datchi ${ }^{24}$ extended the determination to $9 \mathrm{GPa}$ and $900 \mathrm{~K}$ using resistive heating in a diamond anvil cell (DAC). The melting temperature increases in a monotonous fashion with pressure and is well fitted by the Simon-Glatzel equation ${ }^{25}$, typical for weakly-bonded molecular systems. In 2012, Ojwang 


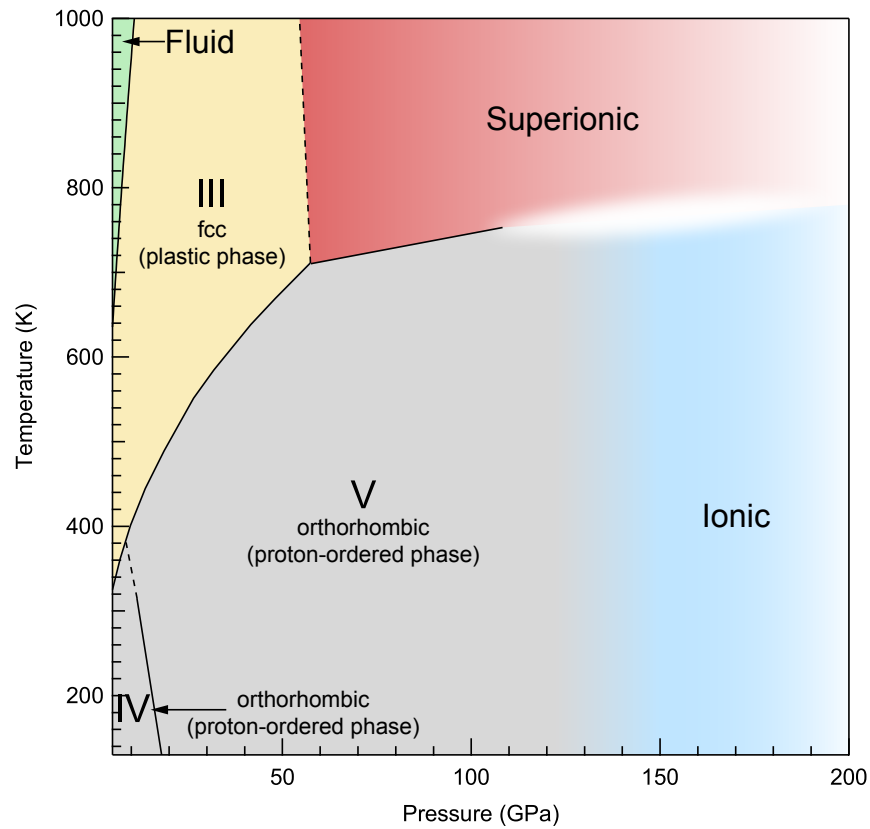

FIG. 1. Phase diagram of ammonia for $P>5 \mathrm{GPa}$, adapted from Ref. 13. The green region correspond to the molecular fluid. The yellow and grey regions correspond to the molecular solid phases composed of $\mathrm{NH}_{3}$ units. The blue region represents the stability domain of the ionic phase reported by Refs. 13 and 14, and the red region, that of the superionic phase.

et al. ${ }^{26}$ reported melting points up to $60 \mathrm{GPa}$ obtained by Raman spectroscopy in the laser-heated DAC. According to this study, the melting temperature reaches a maximum of about $2000 \mathrm{~K}$ at $37 \mathrm{GPa}$ and then decreases. This turnover of the melting line was not expected from the results of AIMD simulations ${ }^{16,17}$, which in turn predict a net increase in the slope of the melting line beyond the triple point joining $\mathrm{NH}_{3}$-III, the superionic solid and the liquid. Ojwang et al. ${ }^{26}$ also reported previously unobserved solid phases of $\mathrm{NH}_{3}$ when quenched from high temperatures, and found that $\mathrm{NH}_{3}$ is chemically unstable at high P-T and partly dissociates into $\mathrm{N}_{2}$ and $\mathrm{H}_{2}$.

In this work, we used the $\mathrm{CO}_{2}$ laser-heated DAC technique combined with in situ Raman spectroscopy and $\mathrm{x}$-ray diffraction (XRD) to investigate the melting line, solid structures and chemical stability of ammonia up to pressures of $40 \mathrm{GPa}$ and temperatures of $3000 \mathrm{~K}$. Unlike the previous work ${ }^{26}$, the ammonia samples here are directly heated by the $\mathrm{CO}_{2}$ laser, thus preventing from chemical reactions that may occur when using a metallic absorber. We also used advanced filtering techniques for x-ray diffraction in the DAC in order to detect the appearance of the weak and the broad liquid signal at melting. Our x-ray based melting points of $\mathrm{NH}_{3}$ agree well with the extrapolation of the melting data of Ninet and Datchi ${ }^{24}$ and do not present the turnover reported by Ojwang et al. ${ }^{26}$. We also do not find evidence for the new solid structures observed by Ojwang et al. ${ }^{26}$

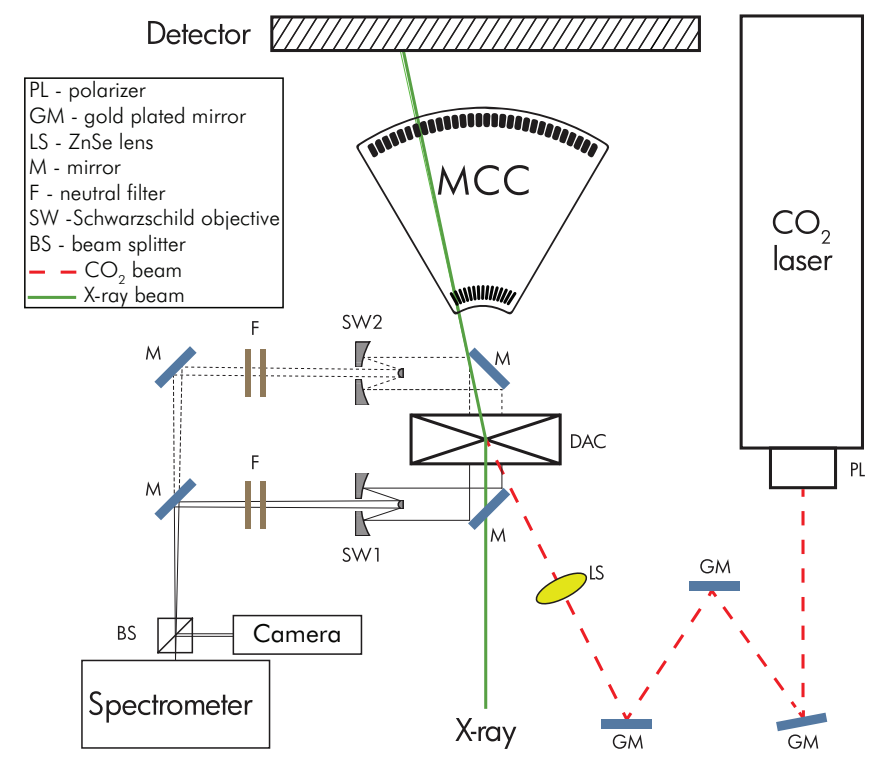

FIG. 2. Schematic of the experimental setup at the ID27 beamline of the ESRF. The sample is heated from one side by a $\mathrm{CO}_{2}$ laser $(\lambda=10.6 \mu \mathrm{m})$. A polarizer is used to finetune the laser power. A $\mathrm{ZnSe}$ lens produces a heating spot of about $40 \mu \mathrm{m}$, which is 10 times as large as the width of the x-ray beam. The temperature is measured by pyrometry using reflective objectives. The MCC is interposed between the sample and the detector to filter out a large part of the Compton scattering from the diamond anvils.

upon quenching from high temperatures. Raman spectroscopy measurements show that liquid ammonia increasingly dissociates into $\mathrm{N}_{2}$ and $\mathrm{H}_{2}$ as temperature is raised at pressures below $10 \mathrm{GPa}$, but remains more stable than the dissociated elements in the range 20-40 GPa below $3000 \mathrm{~K}$.

\section{METHODS}

Ammonia samples (99.99\%, Air Liquide) were loaded cryogenically into a membrane diamond anvil cell using Boehler-Almax designed diamond anvils of $200 \mu \mathrm{m}$ or $300 \mu \mathrm{m}$ culets and $70^{\circ} \mathrm{x}$-ray aperture. A Rhenium foil of $0.2 \mathrm{~mm}$ thickness was used as gasket and was indented to a thickness of 25 to $40 \mu \mathrm{m}$ before drilling holes of $\sim$ $100 \mu \mathrm{m}$. The hole was lined by a gold ring of thickness about $5 \mu \mathrm{m}$ in order to prevent the diffusion of hydrogen into the Re gasket at high temperature ${ }^{27}$. This gold liner also served as pressure sensor in the x-ray diffraction experiments. No thermal insulation layer was used between the diamond anvils and the ammonia sample, in order to avoid chemical reactions.

Angular dispersive x-ray diffraction experiments were performed at the ID27 beamline of the European Synchrotron Radiation Facility (ESRF, Grenoble, France), and Raman scattering experiments were performed at the CEA laboratory. The set-ups for laser heating and tem- 

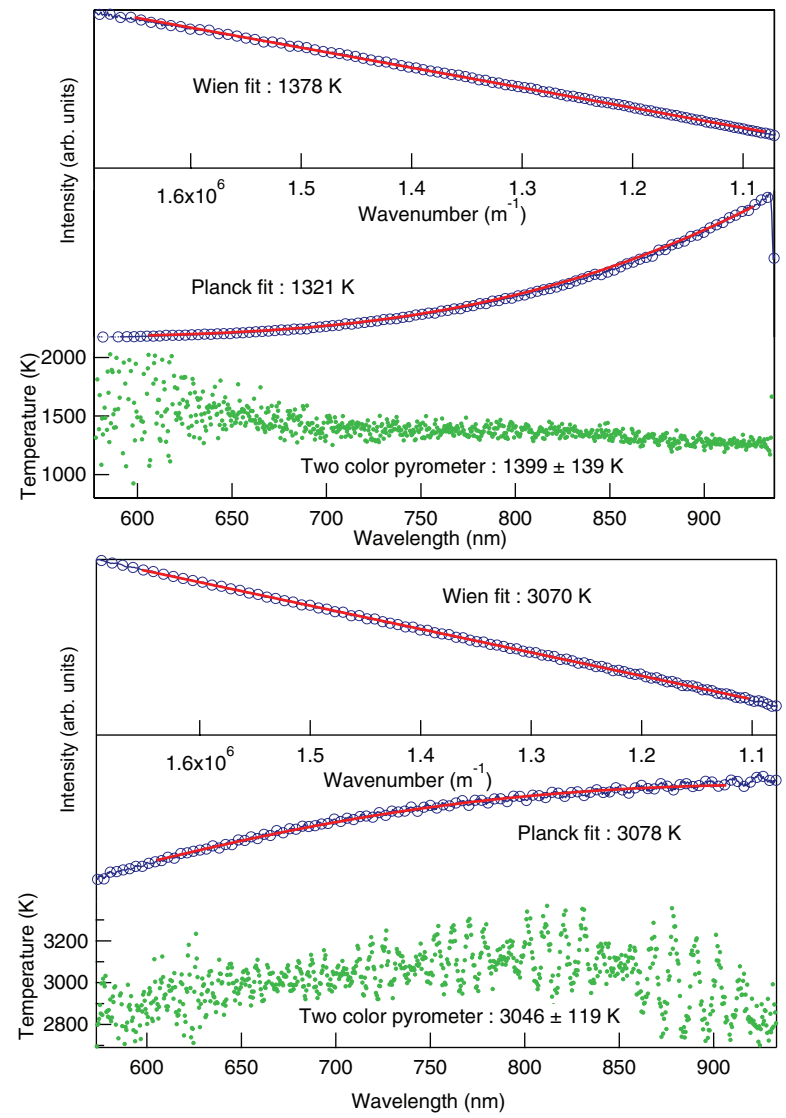

FIG. 3. Examples of measured thermal emission spectra from ammonia samples. The blue circles are experimental data, and the red line are fits to either the Wien (top) or the Planck (middle) distributions. The green dots show the two-color temperature as defined in Ref. 28. The best-fit temperature in each case is indicated. The temperatures reported in this study are those obtained from the Planck distribution and the error bars are taken from the two-color distribution.

perature measurements are very similar for both experiments. A sketch of the experimental setup used at ID27 is shown in Fig. 2. The ammonia samples were heated by a $200 \mathrm{~W} \mathrm{CO}_{2}$ laser (model f201 by Synrad) focussed by a $\mathrm{ZnSe}$ lens to a spot of about $40 \mu \mathrm{m}$. Ammonia directly absorbs the $10.6 \mu \mathrm{m}$ of the $\mathrm{CO}_{2}$ laser, thus no metallic absorber is needed as opposed to lasers emitting at about $1 \mu \mathrm{m}$. As a matter of fact, tests performed using a YAG laser and absorbers made of boron-doped diamond and platinum showed that ammonia either reacts with the absorber, in the case of B-doped diamond, or more easily dissociates, as in the case of $\mathrm{Pt}$ (see Supplemental Material ${ }^{29}$ ). The $\mathrm{CO}_{2}$ laser power can be adjusted in $0.5 \%$ increment using the laser control unit. At ID27, a polarizer was installed at the exit port of the laser for a finer control of the output power. Temperature was determined by pyrometry: the thermal radiation emitted from a $3 \mu \mathrm{m}$-diameter area at the center of the laserheated spot is collected by achromatic Schwarzschild objectives and spectrally analyzed. The typical exposure time ranged from $100 \mathrm{~ms}$ at $3000 \mathrm{~K}$ to $30 \mathrm{~s}$ at $1300 \mathrm{~K}$. We report the temperatures obtained by fitting a Planck distribution to the spectra between 600 and $900 \mathrm{~nm}$. Data points with poor quality fits were rejected. Following the recommendations of Ref. 28, the reported uncertainties are obtained by the sliding two-color pyrometry. Fig. 3 shows typical examples of temperature measurements.

At ID27, the x-ray beam of wavelength $0.3738 \AA$ was focussed to a spot of $3 \times 3 \mu \mathrm{m}$ FWHM and the diffracted signal was collected by a bidimensional MAR CCD detector. The sample-detector distance and x-ray beam position were calibrated with a $\mathrm{CeO}_{2}$ NIST standard powder. The 2D images were integrated with the Dioptas software $^{30}$. Pressure was determined from the volume measurement of gold using the equation of state of Anderson et al. ${ }^{31}$ before and after each heating run, and the difference was included in error bars (see supplementary material for lattice parameters). No correction for thermal pressure has been made. No other pressure calibrant was used to avoid chemical reaction with $\mathrm{NH}_{3}$. To make sure that the $\mathrm{x}$-ray and temperature diagnostics were made on the same part of the sample, particular attention was paid to position the x-ray beam and the input pinhole of the spectrometer for temperature measurement at the center of the heating spot. For light compounds like ammonia in the DAC, a large part of the scattered x-rays comes from the incoherent Compton scattering coming from the diamond anvils. This large background makes it very difficult to observe the very weak diffuse scattering from liquid ammonia. To reduce the diamond Compton contribution, we used a multichannel collimator system (MCC) as described in Refs. 32 and 33. This device spatially isolates a diffracted volume centered on the sample using two concentric sets of slits positioned between the sample and the detector. The counterpart of using the MCC is a signal loss, which required to set the exposure time at $300 \mathrm{~s}$.

Raman spectra were measured using the $488 \mathrm{~nm}$ radiation of an Ar-Kr laser and a $0.5 \mathrm{~m}$ focal length spectrograph equipped with 600 and 1200 lines/mm gratings. The laser beam was focussed to a spot of about $2 \mu \mathrm{m}$ by a $20 \times$ microscope objective (Mitutoyo) and carefully positioned at the center of the heating spot. The backscattered light was collected by the same objective and spatially filtered by confocal optics. The depth of field of this setup is about $7 \mu \mathrm{m}$. The $\mathrm{CO}_{2}$ heating laser enters from the opposite side of the Raman optics at an incidence angle of $\sim 25^{\circ}$. Temperature was measured using the same reflective optics as at ID27. In the first Raman experiments, the temperatures below $\sim 2000 \mathrm{~K}$ could not be measured due to insufficient collection of thermal radiation. Temperatures were then estimated from a linear extrapolation of the temperature versus laser power. Such estimated temperatures will be indicated below by the use of brackets. The setup was later modified to enable a better collection of the thermal radiation. As for x-ray experiments, no pressure calibrant was loaded with the sample and pressure was estimated from the Raman 
shift of the diamond anvil culet using an arithmetic average of the calibrations reported in Refs. 34-37.

\section{RESULTS}

\section{A. X-ray diffraction experiments}

The present x-ray diffraction experiments were performed between 12 to $40 \mathrm{GPa}$ and in the temperature range from 900 to $3500 \mathrm{~K}$. At each pressure, the temperature was increased in several steps at which diffraction patterns were measured. At room temperature the diffraction patterns only contained Bragg reflections from the $P 2_{1} 2_{1} 2_{1}$ solid phases IV and V, as expected. Additional Bragg peaks from phase III were observed as soon as the $\mathrm{CO}_{2}$ laser was turned on, as the temperature of the heated sample rose above the IV-III transition line which is below $650 \mathrm{~K}$ for $P<40 \mathrm{GPa}^{15}$. The persistence of phase IV/V Bragg peaks comes from the axial thermal gradient: since no thermal insulation is used between the diamond anvils and the sample, the part of the sample in contact with the diamonds remains colder than the central region.

The melting criterion is the appearance upon temperature increase of a diffuse scattering ring in the $x$-ray $2 \mathrm{D}$ image coming from the liquid part of the sample, which appears as a broad oscillation on the 1D integrated pattern (Fig. 4). As shown in Fig. 4(b), the diffuse ring appears more clearly after subtraction of a x-ray pattern collected below the melting temperature, as this removes the non-filtered Compton contribution from both the diamond anvils and the sample. Above the melting temperature, the liquid signal increases with temperature as a consequence of the growing amount of melted sample in the heating spot. The melting temperature is taken as the average between the lowest temperature at which the liquid signal is observed and the highest temperature at which only solid diffraction is observed. The error bars reported in Fig. 5 include both the temperature interval within which melting is observed and the uncertainties in temperature measurements.

In total, we have measured 7 melting points up to 39 GPa which are plotted in the phase diagram of Fig. 5, together with the previous melting data of Ninet and Datchi ${ }^{24}$ and Ojwang et al. ${ }^{26}$. Within uncertainties, our melting points agree very well with the extrapolation of the Simon-Glatzel equation fitted to the data of Ninet and Datchi ${ }^{24}$ up to $9 \mathrm{GPa}$, which writes as :

$$
P_{m}\left(T_{m}\right)=0.307+1.135(51)\left[\left(T_{m} / 217.34\right)^{1.510(31)}-1\right],
$$

with $P_{m}$ in GPa and $T_{m}$ in $\mathrm{K}$. The melting temperature thus presents a monotonous increase and does not exhibit a turnover as reported by Ojwang et al. ${ }^{26}$.

Some diffraction patterns collected at high temperatures show the presence of peaks that cannot be assigned to phase III and/or IV(V) of $\mathrm{NH}_{3}$. One example is shown
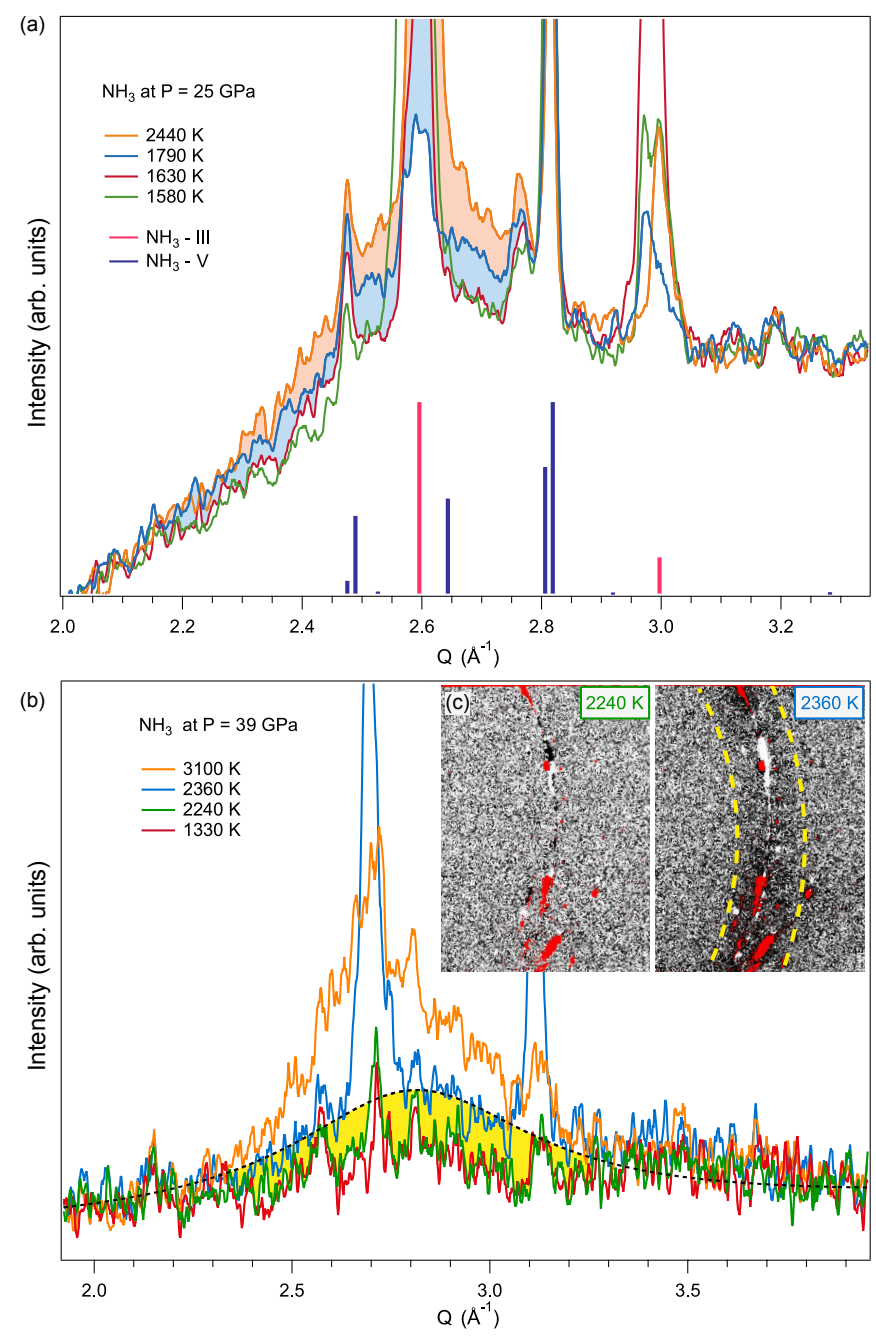

FIG. 4. X-ray diffraction patterns collected at several temperatures below and above melting at (a) $25 \mathrm{GPa}$ and (b,c) $39 \mathrm{GPa}$. In (a), the blue and orange colored areas emphasize the signal from liquid ammonia. Diffraction peaks from solid ammonia III and V still remain present above the melting temperature due to the thermal gradients and their positions are shown by the vertical bars. In (b), the diffraction patterns are obtained by subtracting the image plate at $990 \mathrm{~K}$ and partial masking of the solid peaks. The black dotted line is a Gaussian fit of the liquid signal at $\mathrm{T}=2360 \mathrm{~K}$ and 39 $\mathrm{GPa}$, and the yellow surface corresponds to the liquid signal. The inset shows the diffraction images of $\mathrm{NH}_{3}$ at $39 \mathrm{GPa}$ for $\mathrm{T}=2240 \mathrm{~K}$ and $\mathrm{T}=2360 \mathrm{~K}$. The dotted yellow arcs delimit the region where the halo of the liquid scattering is located. The red color indicates regions which have been masked before integration.

in Fig. 6 for the diffraction pattern at $39 \mathrm{GPa}$ and 2360 $\mathrm{K}$, where the peaks at $2 \theta$ angles of $8.8,9.2,9.6$ and $10.6^{\circ}$ cannot be indexed by $\mathrm{NH}_{3}-\mathrm{III}$ or $\mathrm{NH}_{3}-\mathrm{V}$. These extra peaks appears as isolated spots on the $2 \mathrm{D}$ images and may be indexed by the $\varepsilon$ structure of solid $\mathrm{N}_{2}$ as seen in Fig. 6. Not all Bragg peaks from $\mathcal{\varepsilon}-\mathrm{N}_{2}$ are observed, which indicates that only a few single crystals are seen by 


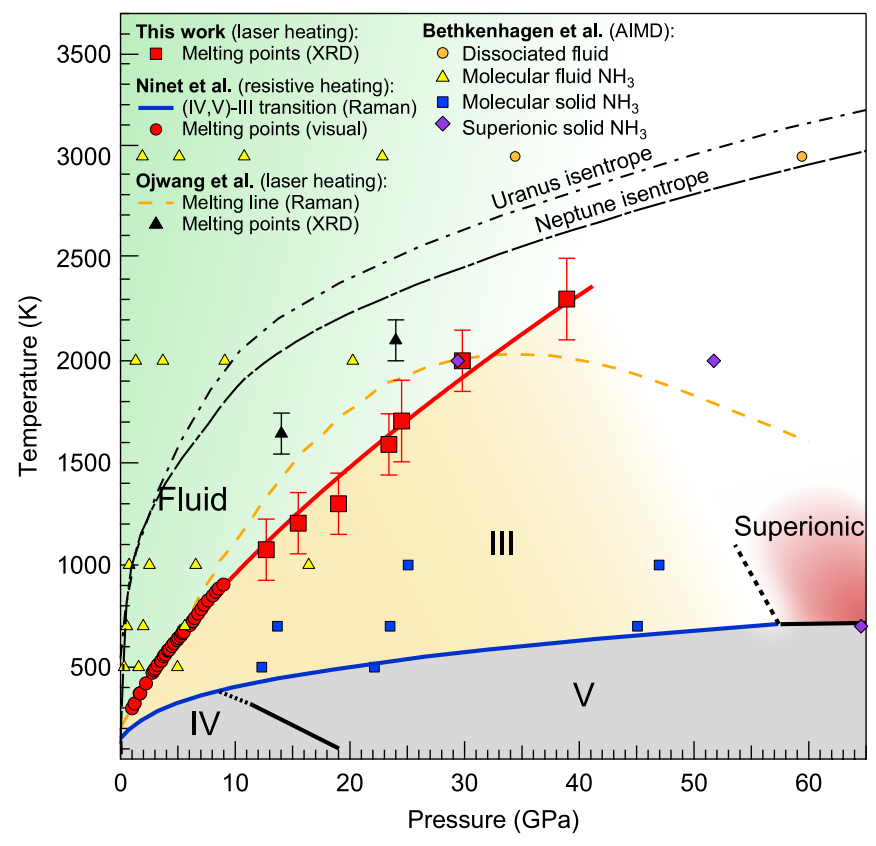

FIG. 5. P-T phase diagram of ammonia. Red squares are the melting points from this work. The red circles correspond to the melting points of Ref. 24. The red solid line is a fit of the Simon-Glatzel law to the melting points of Ninet and Datchi ${ }^{24}$. The orange dashed line is the melting curve of Ojwang et al. ${ }^{26}$ inferred from their Raman study. The black triangles are the x-ray diffraction melting points obtained by the same authors. Orange circles, yellow traingles, blue squares and purple losanges were obtained from the ab initio molecular dynamics simulations of Bethkenhagen et al. ${ }^{17}$, and respectively represent the dissociated fluid, the molecular fluid $\mathrm{NH}_{3}$, the molecular solid $\mathrm{NH}_{3}$ and the superionic solid $\mathrm{NH}_{3}$. The isentropes of Neptune and Uranus are taken from Redmer et al. ${ }^{38}$

the x-ray beam. The reflections from $\mathrm{N}_{2}$ are not observed in the diffraction patterns collected at $300 \mathrm{~K}$ after heating, which could be due to the migration and dispersion of $\mathrm{N}_{2}$ outside the area probed by the x-ray beam when $\mathrm{NH}_{3}$ crystallizes or by a recombination of $\mathrm{N}_{2}$ and $\mathrm{H}_{2}$ into $\mathrm{NH}_{3}$. The appearance of $\mathrm{N}_{2}$ upon heating suggests a partial decomposition of $\mathrm{NH}_{3}$ at high $\mathrm{P}-\mathrm{T}$, as reported by Ojwang et al. ${ }^{26}$. This is further discussed below in the light of our Raman experiments.

We note that the axial thermal gradient in the sample makes it uneasy to extract more quantitative data from the x-ray patterns, such as the volume of the solid phases as a function of pressure and temperature. Indeed, this gradient induces a broadening and an asymmetry of the Bragg peaks resulting from the convolution of peaks at different temperatures. Assigning peak positions to a specific temperature would require the knowledge of the temperature distribution inside the sample, which is not trivial and beyond the scope of this study. Similarly we could not extract the structure factor of the liquid phase as in our previous work on nitrogen ${ }^{39}$ due to the weak

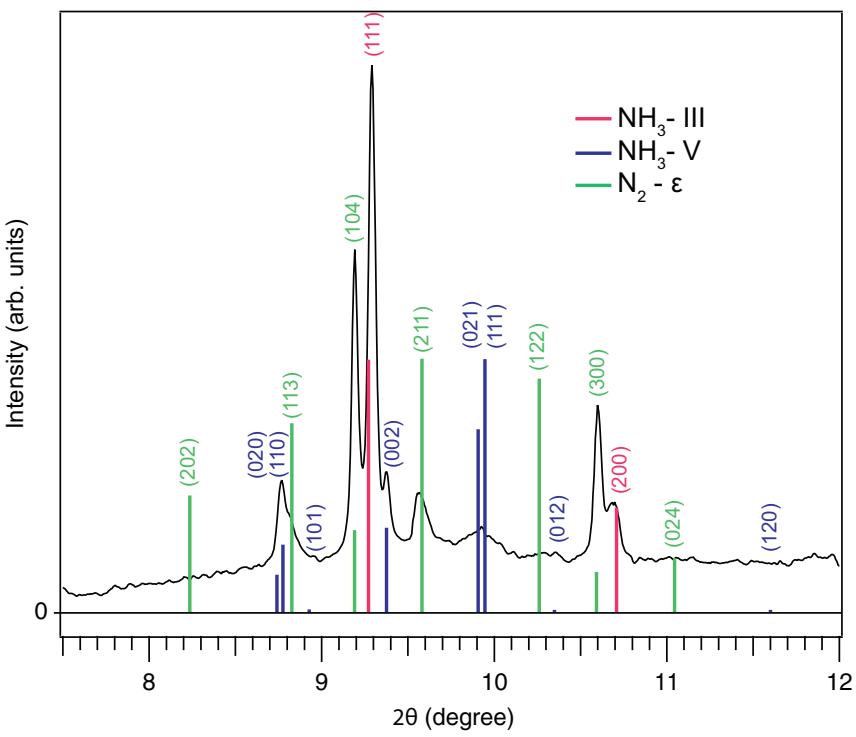

FIG. 6. Diffraction pattern of $\mathrm{NH}_{3}$ at $39 \mathrm{GPa}$ and $2360 \mathrm{~K}$, compared to the simulated patterns of $\mathrm{NH}_{3}-\mathrm{III}, \mathrm{NH}_{3}-\mathrm{V}$ and $\varepsilon-\mathrm{N}_{2}$. The cell parameters obtained by a Le Bail refinement are : $\mathrm{a}=4.0057(82) \AA$ for $\mathrm{NH}_{3}$-III; $\mathrm{a}=2.822(21) \AA, \mathrm{b}=$ $4.898(12) \AA$ and $\mathrm{c}=4.574(13) \AA$ for $\mathrm{NH}_{3}-\mathrm{V} ; \mathrm{a}=7.0124(89)$ $\AA$ and $\mathrm{c}=10.105(21) \AA$ for $\varepsilon-\mathrm{N}_{2}$.

liquid diffuse scattering signal and poor signal-to-noise ratio.

\section{B. Raman spectroscopy experiments}

Raman spectroscopy of ammonia samples under $\mathrm{CO}_{2}$ laser heating were performed from 5 to $40 \mathrm{GPa}$. As for $\mathrm{x}$-ray experiments, the temperature was risen in several steps at a fixed load. The goals of these experiments were (1) to see whether melting can be detected through the changes in the Raman spectrum, and (2) to investigate the chemical stability of $\mathrm{NH}_{3}$ at high $\mathrm{P}-\mathrm{T}$.

We recall that the three solid phases of $\mathrm{NH}_{3}$ observed between 5 and $40 \mathrm{GPa}$ are the proton-ordered solid phases IV and V, and the proton-disordered phase III. The Raman spectrum of the orthorhombic solid phases $\mathrm{IV} / \mathrm{V}$ of $\mathrm{NH}_{3}$ is composed of 21 lattice phonons below $800 \mathrm{~cm}^{-1}, 4 v_{2}$ and $8 v_{4}$ bending modes at around 1200 $\mathrm{cm}^{-1}$ and $1650 \mathrm{~cm}^{-1}$ respectively, and $4 v_{1}+8 v_{3}$ stretching modes located in the frequency window 3100-3500 $\mathrm{cm}^{-1}$ (Ref. 40). The stretching band is the more intense and also includes overtones of $v_{4}$ in Fermi resonance with $v_{3}$ modes. In the high-temperature cubic phase III, there is no Raman-active lattice modes and the experimental Raman spectrum only displays a broad $\mathrm{N}-\mathrm{H}$ stretching band with overlapping peaks from $v_{1}, v_{3}$ and $2 v_{4}$ vibrations $^{41,42}$.

When trying to determine melting with Raman spectroscopy, the first criterion to be considered is the disappearance of the lattice modes which only exist in the 



FIG. 7. (a) Evolution of the low frequency Raman spectra of $\mathrm{NH}_{3}$ with temperature at 31 GPa. From the bottom to the top spectrum, the power of the $\mathrm{CO}_{2}$ laser is gradually increased to ramp up the temperature. The lattice modes of $\mathrm{NH}_{3}-\mathrm{IV}$ are visible at $300 \mathrm{~K}$ and gradually disappear with $\mathrm{T}$ due to the transition to phase III. The temperatures between 300 and $820 \mathrm{~K}$ could not be measured due to insufficient thermal emission. (b) Comparison between the N-H stretch Raman band in the solid $(\mathrm{T}=1470 \mathrm{~K})$ and liquid $(\mathrm{T}=2400 \mathrm{~K})$ phases at $35 \mathrm{GPa}$. The black lines are the experimental data, and the blue and red lines are fits using Gaussian peaks for the solid and liquid, respectively. The dotted lines show the decomposition into Gaussian peaks, offset for visibility. The fit residuals are shown in the top plot.

crystalline phase. Unfortunately, the stable solid phase before melting is $\mathrm{NH}_{3}$-III which has no Raman-active lattice modes. Fig. 7(a) shows the evolution with temperature of the low frequency Raman spectra of ammonia at $31 \mathrm{GPa}$. The lattice modes of $\mathrm{NH}_{3}-\mathrm{V}$ are clearly observed at room temperature, and gradually disappear as temperature increases. At $820 \mathrm{~K}$, the lowest measurable temperature by pyrometry, all peaks have disappeared due to the transition to phase III. The temperature of $820 \mathrm{~K}$ is higher than the IV-III transition determined by resistive heating at this pressure $(580 \mathrm{~K}$, according to Ref. 15). This is because part of the sample seen by the Raman probe is still in phase IV due to the axial thermal gradient. At this pressure, $\mathrm{NH}_{3}$ melts at $1970 \mathrm{~K}$ according to our x-ray study, and it can be seen from Fig. 8 that there is no significant change in the low-frequency Raman spectra across melting.

In Ojwang et al. ${ }^{26}$, the authors used as criterion for melting the change in shape of the $\mathrm{N}-\mathrm{H}$ stretch band. According to them, the stretching modes all merge in a broad single band on melting. In Fig. 7(b), we compare the Raman $\mathrm{N}-\mathrm{H}$ stretch band measured at $35 \mathrm{GPa}$ at two temperatures, $1470 \mathrm{~K}$ and $2400 \mathrm{~K}$. At this pressure, the melting temperature of $\mathrm{NH}_{3}$ measured in the present work is close that reported in Ojwang et al. ${ }^{26}$ and is about $2000 \mathrm{~K}$. We note that the temperature gra- dient over the sample thickness which is probed by our Raman setup may be estimated from the difference in temperature between the observed V-III transition (820 $\mathrm{K}$, see above) and actual one $(580 \mathrm{~K})$, that is $240 \mathrm{~K}$. At $2400 \mathrm{~K}$, we may thus be confident that the collected spectra mainly comes from the hot liquid phase, which in addition scatters more than the colder solid. It can be observed in Fig. 7(b) that the $\mathrm{N}-\mathrm{H}$ stretch bands at $1470 \mathrm{~K}$ (solid) and $2400 \mathrm{~K}$ (liquid) are very similar in shape, and that both present a structure which can be decomposed into several peaks as indicated by the fits shown in the figure. We thus conclude that the change in shape of the $\mathrm{N}-\mathrm{H}$ stretch band is not a valid criterion for melting. The similarity in Raman spectra between the solid phase III and the liquid was previously noted at low pressures ${ }^{42}$.

As seen in Fig. 8, the Raman spectra collected at high temperatures show the appearance of two new peaks around $2340 \mathrm{~cm}^{-1}$ and $4260 \mathrm{~cm}^{-1}$, which can be assigned to the stretching vibrations of the $\mathrm{N}_{2}$ and $\mathrm{H}_{2}$ molecules, respectively. This suggests that a partial decomposition of $\mathrm{NH}_{3}$ occurs at high temperatures, which is consistent with our x-ray diffraction observations. In the run at 6 $\mathrm{GPa}$, the hydrogen vibron was detected at an estimated temperature of $1300 \mathrm{~K}$, while the nitrogen vibron started to be observed at $1470 \mathrm{~K}$. With increasing temperature, 
the $\mathrm{N}_{2}$ and $\mathrm{H}_{2}$ vibrons broaden and shift to lower frequencies as expected. Above $\sim 2000 \mathrm{~K}$, two more $\mathrm{N}_{2}$ and one more $\mathrm{H}_{2}$ vibron appear on the low frequency side of the principal modes, whose intensities increase with temperature (Fig. 8(a)). The frequencies of these additional vibrons well match those of the vibrational transitions from excited states of hot $\mathrm{N}_{2}$ and hot $\mathrm{H}_{2}$ reported in Ref. 43.

The Raman spectra collected at $6 \mathrm{GPa}, 300 \mathrm{~K}$ after heating, outside and inside the laser heated zone, respectively, show that only the heated zone contains $\mathrm{N}_{2}$ and $\mathrm{H}_{2}$. The frequencies of the $\mathrm{N}_{2}$ and $\mathrm{H}_{2}$ vibrons are $v_{2}\left(\mathrm{~N}_{2}\right)$ $=2341.7 \mathrm{~cm}^{-1}$ and $v\left(\mathrm{H}_{2}\right)=4234.5 \mathrm{~cm}^{-1}$, respectively. These are close to the reported frequencies for pure solid $\mathrm{N}_{2}\left(v_{2}\left(\mathrm{~N}_{2}\right)=2342.8 \mathrm{~cm}^{-1}\right.$ from Ref. 44) and pure solid $\mathrm{H}_{2}\left(v\left(\mathrm{H}_{2}\right)=4232 \mathrm{~cm}^{-1}\right.$ from Ref. 45), which suggests that $\mathrm{NH}_{3}, \mathrm{~N}_{2}$, and $\mathrm{H}_{2}$ are phase separated.

Above $20 \mathrm{GPa}$, the signature of a partial decomposition is also observed, as depicted in Fig. 8(b) at $31 \mathrm{GPa}$, but unlike for lower pressures, the intensities of the $\mathrm{N}_{2}$ and $\mathrm{H}_{2}$ peaks remain very low and do not increase with temperature. The hot bands of $\mathrm{N}_{2}$ and $\mathrm{H}_{2}$ are not observed at this pressure either. As at lower pressure, the decomposition is irreversible, as the $\mathrm{N}_{2}$ and $\mathrm{H}_{2}$ peaks remain present at the heating spot when reverting to room temperature. The frequencies of the vibrational modes $v_{2}\left(\mathrm{~N}_{2}\right)$ and $v\left(\mathrm{H}_{2}\right)$ at $300 \mathrm{~K}$ are again consistent with the frequencies of solid $\mathrm{N}_{2}{ }^{46}$ and solid $\mathrm{H}_{2}{ }^{45}$.

\section{DISCUSSION}

As seen above, the present x-ray experiments show that the melting temperature of ammonia steadily increases up to $39 \mathrm{GPa}$ without showing any evidence of a sudden change in slope or inflection. The present data agree within uncertainties with the extrapolation of the melting data of Ninet and Datchi ${ }^{24}$. We note that the presence of $\mathrm{N}_{2}$ and $\mathrm{H}_{2}$ impurities in the sample does not seem to alter the melting temperature of $\mathrm{NH}_{3}$ at a level which can be detected by present experiments. This is consistent with the fact that, according to our Raman measurements, the decomposition of $\mathrm{NH}_{3}$ occurs in the liquid phase, and that only a very small amount of $\mathrm{N}_{2}$ and $\mathrm{H}_{2}$ was observed above $20 \mathrm{GPa}$ and below $3000 \mathrm{~K}$.

Ojwang et al. ${ }^{26}$ 's melting temperatures are 200 to 300 $\mathrm{K}$ higher than present ones below $30 \mathrm{GPa}$, and their melting line turnover at $37 \mathrm{GPa}$ is not confirmed by our measurements. This difference is likely explained by the fact that the Raman melting criterion used by these authors is not robust enough, as shown in Section III B. Ojwang et al. ${ }^{26}$ also reported two melting points at 14 and 24 GPa obtained from x-ray experiments, where they use the same criterion of melting as in the present work, that is the appearance of the diffuse signal from the liquid. The two measured melting temperatures, shown on the Fig. 5 , are $\sim 500 \mathrm{~K}$ higher than the present data at the same pressure. This overestimation likely results from the fact that the liquid signal was too weak to be detected at lower temperatures, for the amount of liquid was too small. Indeed in our measurements taken without the MCC, we found that the signal-to-noise ratio is much too low to discern the signal of the fluid at melting. A larger quantity of fluid would then be required, as obtained by increasing the temperature of the hot spot, which is mistakenly interpreted as a higher melting temperature.

Fig. 5 compares the experimental phase diagram of $\mathrm{NH}_{3}$ compiled form present and previous works to the predictions of the AIMD simulations of Bethkenhagen et al. ${ }^{17}$. Below $2000 \mathrm{~K}$, these simulations predict that fluid $\mathrm{NH}_{3}$ is molecular and solidifies into the molecular phase III. The melting pressures appear larger than the experimental ones, which is also the case for the AIMD work of Cavazzoni et al. ${ }^{16}$ (see also Ref. ${ }^{24}$ ). At $2000 \mathrm{~K}$, DFT predicts that the fluid cristallizes into the superionic solid between 20 and $28 \mathrm{GPa}$, which this time is smaller than the experimental melting point (29 GPa). These simulations also suggest a kink on the melting line in the range 20-30 GPa, 1000-2000 K resulting from the triple point between the molecular fluid, the molecular solid and the superionic solid, which our experiments do not observe within uncertainties.

An interesting output of this study is that the melting temperature of $\mathrm{NH}_{3}$ increases more rapidly with pressure than the isentropes of Neptune and Uranus do (see Fig. 5). An extrapolation of our melting data suggests that the melting line crosses these isentropes at around 55 and $65 \mathrm{GPa}$ for Neptune and Uranus respectively, pressure beyond which ammonia would occur as a solid phase in these planets. On contrary, the AIMD simulations of Ref. 17 predict that above $3000 \mathrm{~K}$ the melting line become very flat and never crosses the planetary isentropes. We note that it is unlikely to find pure ammonia inside Neptune and Uranus, but rather mixed with the major component $\mathrm{H}_{2} \mathrm{O}$ and other elements. DFT calculations indicate that the slope of the melting curve of water steeply increase above the triple point between the molecular fluid, the molecular solid and the superionic solid. ${ }^{38}$ As a result, the melting temperature of $\mathrm{H}_{2} \mathrm{O}$ grows larger than the planetary isentropes above ca. 100 GPa. AIMD simulations of the 1:1 mixture of $\mathrm{H}_{2} \mathrm{O}$ and $\mathrm{NH}_{3}$ also predict a crossing of the melting line and the planetary isentropes. ${ }^{47}$ These results would thus indicate that a solid layer of mixed superionic ice could exist in the interior of Neptune and Uranus.

The partial decomposition of $\mathrm{NH}_{3}$ at high $\mathrm{P}-\mathrm{T}$ is confirmed by the present study. Since our samples are purely composed of ammonia, we may conclude that this decomposition is an intrinsic effect of elevated temperatures. Indeed, the decomposition is unlikely to be catalyzed by the diamond anvils for the following reasons: first, the sample part in contact with the anvils cannot be much hotter than a few hundred $\mathrm{K}$ because the heat is very efficiently transported away by the anvils. In our previous diamond anvil cell experiments using resistive heat- 

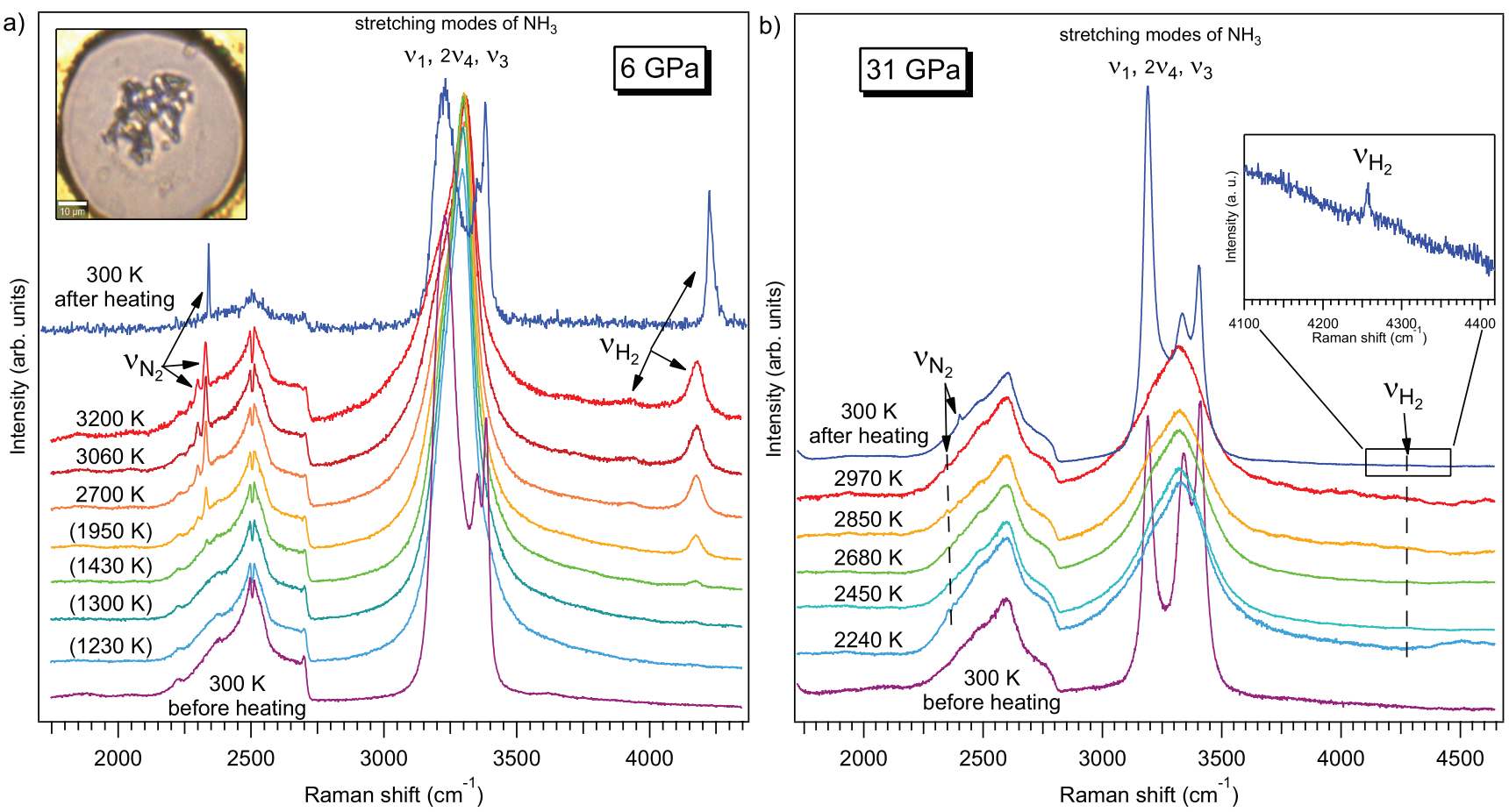

FIG. 8. Evolution with temperature of the Raman spectra of $\mathrm{NH}_{3}$ at (a) 6 GPa and (b) 31 GPa. In both cases, the purple curves show the initial spectrum from a freshly loaded sample before heating. The $\mathrm{N}-\mathrm{H}$ stretching band, composed of $\nu_{1}, \nu_{3}$ and overtones of $\nu_{4}$ modes, occurs in the frequency window 3100-3500 $\mathrm{cm}^{-1}$. The band between 2250 and $2750 \mathrm{~cm}^{-1}$ is the second order Raman band of diamond. Upon heating the $\mathrm{N}_{2}$ and $\mathrm{H}_{2}$ vibron peaks appear at about $2340 \mathrm{~cm}^{-1}$ and $4260 \mathrm{~cm}^{-1}$, respectively. Additional hot vibrational bands of $\mathrm{N}_{2}$ and $\mathrm{H}_{2}$ appear at higher tempeartures as indicated by the arrows. In (a), the temperatures indicated in brackets are estimated from the laser power. After a stepwise return to $300 \mathrm{~K}$, the principal $\mathrm{N}_{2}$ and $\mathrm{H}_{2}$ vibrons remain as seen in the blue spectrum in (a) and (b). The inset of (a) shows a photograph of the sample after temperature quench. The darker region is where the sample was heated and contains inclusions of $\mathrm{N}_{2}$ and $\mathrm{H}_{2}$ solids.

ing ${ }^{15,24}$ where both the sample and anvils are at high temperature, we did not see any evidence of decomposition of ammonia up to $900 \mathrm{~K}$. Second, we did not observe any chemical attack of the anvils surface after the experiments. Third, if carbon was released in the bulk of the sample, it would easily form hydrocarbons such as methane by reaction with the hot hydrogen, and we did not observe it either by Raman or XRD. At $6 \mathrm{GPa}$, $\mathrm{N}_{2}$ and $\mathrm{H}_{2}$ were detected at temperatures above $1300 \mathrm{~K}$, which is well above the melting point $(700 \mathrm{~K})$ at this pressure. This is consistent with the fact that no sign of these species were previously observed ${ }^{24}$ in the solid up to the melting temperature. The Raman signal of both $\mathrm{N}_{2}$ and $\mathrm{H}_{2}$ increased in intensity with temperature, indicating that the equilibrium between $\mathrm{NH}_{3}, \mathrm{~N}_{2}$ and $\mathrm{H}_{2}$ balances more strongly towards the dissociated elements with increasing temperature. This fairly agrees with the free energy calculations of Ojwang et al. ${ }^{26}$, which predict that $\mathrm{NH}_{3}$ is unstable with respect to the $\mathrm{N}_{2}+\mathrm{H}_{2}$ mixture at $8 \mathrm{GPa}$ and $700 \mathrm{~K}$. The AIMD simulations of Bethkenhagen et al. ${ }^{17}$ also predicted that below 20 $\mathrm{GPa}$, fluid ammonia decomposes into $\mathrm{N}_{2}$ and $\mathrm{H}_{2}$; however the temperature range (4000-5000 K) at which decomposition is observed is higher than in present experiments, which could be due to the limited time ( 10 ps) during which these simulations are performed. Above $20 \mathrm{GPa}$, the amount of $\mathrm{N}_{2}$ and $\mathrm{H}_{2}$ formed upon heating appears to be smaller than at lower pressures, and stay stable up to $\sim 3000 \mathrm{~K}$. This indicates that ammonia is more stable than the dissociated elements from 20 to $40 \mathrm{GPa}$, up to at least $3000 \mathrm{~K}$. We note that the Raman signal of $\mathrm{N}_{2}$ observed in the experiments of Ojwang et al. ${ }^{26}$ seems more intense in this pressure range. This difference could be due to the diffusion of $\mathrm{H}_{2}$ into the metallic Ir coupler or Re gasket in the previous work, which would unbalance the chemical equilibrium $2 \mathrm{NH}_{3} \rightleftharpoons \mathrm{N}_{2}+3 \mathrm{H}_{2}$ towards decomposition. In present experiments, diffusion is more limited, as no coupler is present and a gold ring separates the sample from the Re gasket, which acts as a diffusion barrier for $\mathrm{H}_{2}{ }^{27}$.

Apart from the occurrence of $\mathrm{N}_{2}$ and $\mathrm{H}_{2}$ at high $\mathrm{T}$, we did not find evidence for new phases of $\mathrm{NH}_{3}$ or other species in the present Raman and XRD data after annealing. This also contrasts with the study of Ojwang et al. ${ }^{26}$ who reported various phases differing in Raman spectra from phases IV and $\mathrm{V}$ when quenching the high $\mathrm{T}$ liquid to room temperature above $5 \mathrm{GPa}$. These "new" phases appeared after a very intense and sudden light emission (or "flash") was observed during the laser heating. We did not observe such "flashes" in our experiments 
with $\mathrm{CO}_{2}$ laser heating, which suggests that they may be the result of a direct coupling between the sample and the YAG laser used by Ojwang et al. ${ }^{26}$. This coupling may either generate a rapid and large temperature increase, and thus an intense thermal emission, or induce a strong luminescence. Such a strong luminescence was observed in laser heating experiments of compressed $\mathrm{O}_{2}$ below $50 \mathrm{GPa}$, where it was suggested to originate from photoinduced ionic species ${ }^{48}$. This could also be the case in ammonia, where ionic species in the hot dense liquid could be either photo-induced by the YAG laser, or pressure-induced as suggested by AIMD simulations ${ }^{16,17}$. The presence of the metallic coupler may also catalyse chemical reactions between ions and/or molecules to form $\mathrm{N}_{x} \mathrm{H}_{y}$ compounds, whose existence at high $\mathrm{P}-\mathrm{T}$ has been recently inferred from experiment ${ }^{49-51}$ and theory ${ }^{52,53}$ and could explain the additional $\mathrm{N}-\mathrm{H}$ Raman bands observed in the quenched samples by Ojwang et al. ${ }^{26}$.

We made several attempts to collect x-ray and Raman data above $40 \mathrm{GPa}$. For all samples compressed at 45 $\mathrm{GPa}$ and above, the diamond anvils failed during laser heating before we could collect data. We suspect that this is related to the occurrence of the superionic solid phase which becomes stable before melting. In the superionic solid indeed, the protons diffuse rapidly through the nitrogen lattice and it is well known that the diffusion of $\mathrm{H}^{+}$into the stressed anvils make them fail at lower pressures than normal. As seen in Fig. 5, the extrapolation of the $\mathrm{NH}_{3}-\mathrm{III} /$ superionic transition line of Ninet et al. ${ }^{15}$ suggests the presence of a triple point near 45 $\mathrm{GPa}$ and $2500 \mathrm{~K}$, which is consistent with our conjecture.

\section{CONCLUSION}

In this work, we presented an experimental investigation of ammonia at high pressures and temperatures using synchrotron x-ray diffraction and Raman spectroscopy. The melting line inferred from our x-ray data shows a continuous increase up to $40 \mathrm{GPa}$, following the extrapolation of the previous melting data of Ninet and Datchi $^{24}$. No turnover of the melting line is observed, at odds with the results of Ojwang et al. ${ }^{26}$. We confirm, based on our Raman measurements, that solid ammonia partly dissociates into $\mathrm{N}_{2}$ and $\mathrm{H}_{2}$, showing that the chemical equilibrium between these three species is a complex function of pressure and temperature. Below $10 \mathrm{GPa}$, the equilibrium balances more strongly towards the diatomic molecules at high temperatures, while at higher pressures, ammonia remains the more stable compound up to $3000 \mathrm{~K}$. The systematic anvil failures above $40 \mathrm{GPa}$ at high temperature may indicate the presence of the triple point between $\mathrm{NH}_{3}$-III, the superionic solid and the liquid at $45 \mathrm{GPa}, 2300 \mathrm{~K}$. Extension of this work to higher P-T conditions would be very interesting to investigate the melting line of the superionic solid and the structural and chemical properties of the dissociated fluid predicted by first-principles calculations, but this will require new technical developments in order to better confine the sample and better detect the liquid scattering.

\section{ACKNOWLEDGMENTS}

We acknowledge the ESRF for provision of beam time under Long-Term Project HD-463, proposals (HC-2185 and $\mathrm{HC}-4074$ ) and the Agence Nationale de la Recherche for financial support under grant ANR 13-BS04-0015 (MOFLEX) and ANR 15-CE30-0008-01 (SUPER-ICES).
* queyroux@protonmail.ch

$\dagger$ frederic.datchi@sorbonne-universite.fr

1 W. B. Hubbard, Science 214, 145 (1981).

2 T. Guillot, Science 286, 72 (1999).

3 D. Sudarsky, A. Burrows, and I. Hubeny, Astrophy. J. 588, 1121 (2003).

${ }^{4}$ O. L. Kuskov and V. A. Kronrod, Icarus 177, 550 (2005).

${ }^{5}$ C. C. Porco, P. Helfenstein, P. C. Thomas, A. P. Ingersoll, J. Wisdom, R. West, G. Neukum, T. Denk, R. Wagner, T. Roatsch, S. Kieffer, E. Turtle, A. McEwen, T. V. Johnson, J. Rathbun, J. Veverka, D. Wilson, J. Perry, J. Spitale, A. Brahic, J. A. Burns, A. D. DelGenio, L. Dones, C. D. Murray, and S. Squyres, Science 311, 1393 (2006).

6 B. I. Olovsson and D. H. Templeton, Acta Crystallogr. 12, 832 (1959).

7 J. W. Reed and P. M. Harris, J. Chem. Phys. 35, 1730 (1961).

8 A. W. Hewat and C. Riekel, Acta Crystallogr. Sect. A 35, 569 (1979).
9 J. Eckert, R. L. Mills, and S. K. Satija, J. Chem. Phys. 81, 6034 (1984).

10 R. B. Von Dreele and R. C. Hanson, Acta Crystallogr. Sect. C 40, 1635 (1984).

11 J. S. Loveday, R. J. Nelmes, W. G. Marshall, J. M. Besson, S. Klotz, and G. Hamel, Phys. Rev. Lett. 76, 74 (1996).

12 S. Ninet, F. Datchi, S. Klotz, G. Hamel, J. S. Loveday, and R. J. Nelmes, Phys. Rev. B 79, 100101(R) (2009).

13 S. Ninet, F. Datchi, P. Dumas, M. Mezouar, G. Garbarino, A. Mafety, C. J. Pickard, R. J. Needs, and a. M. Saitta, Phys. Rev. B 89, 174103 (2014).

14 T. Palasyuk, I. Troyan, M. Eremets, V. Drozd, S. Medvedev, P. Zaleski-Ejgierd, E. Magos-Palasyuk, H. Wang, S. A. Bonev, D. Dudenko, and P. Naumov, Nat. Commun. 5, 3460 (2014).

15 S. Ninet, F. Datchi, and A. M. Saitta, Phys. Rev. Lett. 108, 165702 (2012).

16 C. Cavazzoni, G. L. Chiarotti, S. Scandolo, E. Tosatti, M. Bernasconi, and M. Parrinello, Science 283, 44 (1999). 
17 M. Bethkenhagen, M. French, and R. Redmer, J. Chem. Phys. 138, 234504 (2013).

18 R. D. Dick, J. Chem. Phys. 74, 4053 (1981).

19 A. C. Mitchell, J. Chem. Phys. 76, 6273 (1982).

${ }^{20}$ W. J. Nellis, D. C. Hamilton, N. C. Holmes, H. B. Radousky, F. H. Ree, A. C. Mitchell, and M. Nicol, Science 240, 779 (1988).

${ }^{21}$ H. B. Radousky, A. C. Mitchell, and W. J. Nellis, J. Chem. Phys. 93, 8235 (1990).

22 W. J. Nellis, N. C. Holmes, A. C. Mitchell, D. C. Hamilton, and M. Nicol, J. Chem. Phys. 107, 9096 (1997).

23 R. C. Hanson and M. Jordan, J. Phys. Chem. 84, 1173 (1980).

24 S. Ninet and F. Datchi, J. Chem. Phys. 128, 154508 (2008).

${ }^{25}$ F. Simon and G. Glatzel, Z. Anorg. Allg. Chem. 178, 309 (1929).

26 J. G. O. Ojwang, R. S. McWilliams, X. Ke, and A. F. Goncharov, J. Chem. Phys. 137, 64507 (2012).

27 F. Datchi, P. Loubeyre, and R. LeToullec, Phys. Rev. B 61, 6535 (2000).

${ }^{28}$ L. Benedetti and P. Loubeyre, High Pres. Res. 24, 423 (2004).

29 See Supplemental Material at [url] for more information about chemical reaction between ammonia and absorbers under YAG laser heating.

30 C. Prescher and V. B. Prakapenka, High Pres. Res. 7959, 223 (2015).

31 O. L. Anderson, D. G. Isaak, and S. Yamamoto, J. Appl. Phys. 65, 1534 (1989).

32 M. Mezouar, P. Faure, W. Crichton, N. Rambert, B. Sitaud, S. Bauchau, and G. Blattmann, Rev. Sci. Instrum. 73, 3570 (2002).

${ }^{33}$ G. Weck, G. Garbarino, S. Ninet, D. Spaulding, F. Datchi, P. Loubeyre, and M. Mezouar, Rev. Sci. Instrum. 84, 063901 (2013).

${ }^{34}$ F. Occelli, P. Loubeyre, and R. LeToullec, Nat. Mater. 2, 151 (2003).

35 Y. Akahama and H. Kawamura, J. Appl. Phys. 96, 3748 (2004).
36 M. Popov, J. Appl. Phys. 95, 5509 (2004).

37 B. J. Baer, M. E. Chang, and W. J. Evans, J. Appl. Phys. 104, 34504 (2008).

38 R. Redmer, T. R. Mattsson, N. Nettelmann, and M. French, Icarus 211, 798 (2011).

${ }^{39}$ G. Weck, F. Datchi, G. Garbarino, S. Ninet, J. A. Queyroux, T. Plisson, M. Mezouar, and P. Loubeyre, Phys. Rev. Lett. , 1 (2017).

40 S. Ninet, F. Datchi, A. Saitta, M. Lazzeri, and B. Canny, Phys. Rev. B 74, 104101 (2006).

41 M. Gauthier, P. Pruzan, J. C. Chervin, and A. Polian, Solid State Communications 68, 149 (1988).

42 T. Kume, S. Sasaki, and H. Shimizu, J. Raman Spectrosc. 32, 383 (2001).

43 A. F. Goncharov and J. Crowhurst, Phys. Rev. Lett. 96, 55504 (2006).

44 D. Schiferl, S. Buchsbaurn, and R. L. Mills, J. Phys. Chem. 89, 2324 (1985).

45 I. F. Silvera and R. J. Wijngaarden, Phys. Rev. Lett. 47, 39 (1981).

46 H. Schneider, W. Hafner, A. Wokaun, and H. Olijnyk, J. Chem. Phys. 96, 8046 (1992).

47 M. Bethkenhagen, D. Cebulla, R. Redmer, and S. Hamel, J. Phys. Chem. A 119, 10582 (2015).

48 M. Santoro, E. Gregoryanz, H. kwang Mao, and R. J. Hemley, Solid State Commun. 144, 225 (2007).

49 D. K. Spaulding, G. Weck, P. Loubeyre, F. Datchi, P. Dumas, and M. Hanfland, Nat. Commun. 5, 5739 (2014).

50 A. F. Goncharov, N. Holtgrewe, G. Qian, C. Hu, A. R. Oganov, M. Somayazulu, E. Stavrou, C. J. Pickard, A. Berlie, F. Yen, M. Mahmood, S. S. Lobanov, Z. Konôpková, and V. B. Prakapenka, J. Chem. Phys. 142, 214308 (2015).

51 H. Wang, M. I. Eremets, I. Troyan, H. Liu, Y. Ma, and L. Vereecken, Sci. Rep. 5, 13239 (2015).

52 I. G. Batyrev, J. Phys. Chem. A 121, 638 (2017).

53 G.-R. Qian, H. Niu, C.-H. Hu, A. R. Oganov, Q. Zeng, and H.-Y. Zhou, Sci. Rep. 6, 25947 (2016). 